\title{
Smart Agriculture; Farm Irrigation System Using IoT
}

\author{
Amera I. Badran \\ amera_istiqlal@uomosul.edu.iq \\ Manar Y. Kashmoola \\ manar.kashmola@uomosul.edu.iq \\ Department of Computer Science \\ College of Computer Science and Mathematics \\ University of Mosul, Mosul, Iraq
}

Received on: $27 / 07 / 2020$

Accepted on: 24/08/2020

\section{ABSTRACT}

Due to the increase of development in modern technology which entered in most fields of life including sustainable agriculture; most studies revealed that most lesions result from over irrigation which causes fungi in plant and soil salinity. Recently; some very important terms emerged and changed most agricultural concepts such as the sustainable agriculture, green cities and smart irrigation systems. Most of these systems improved the quality of production and reduced lesions. In this paper a smart irrigation system was designed depending on Field Capacity F.C value, Wilting Point W.P value. In addition to the ranges of moisture that are measured in the field which are important in decision making of irrigation and selecting the best values to rely on such as threshold value in designing for the sake of maintaining moisture in the soil permanently. The best field moisture value was recorded when designing was \%24 at threshold value in a clay soil field. Finally; the best types of microcontrollers ESP8266 \& ESP-32S and moisture sensors, which are used to upload the data to Adafruit server. Also, the fast and light Message Queuing Telemetry Transport (MQTT) protocol, was used to transfer the ranges of moisture through the system and cloud computing.

Keywords: Internet of Things, MQTT protocol, Smart Irrigation, Soil moisture, soil constants, Field capacity, wilting point.

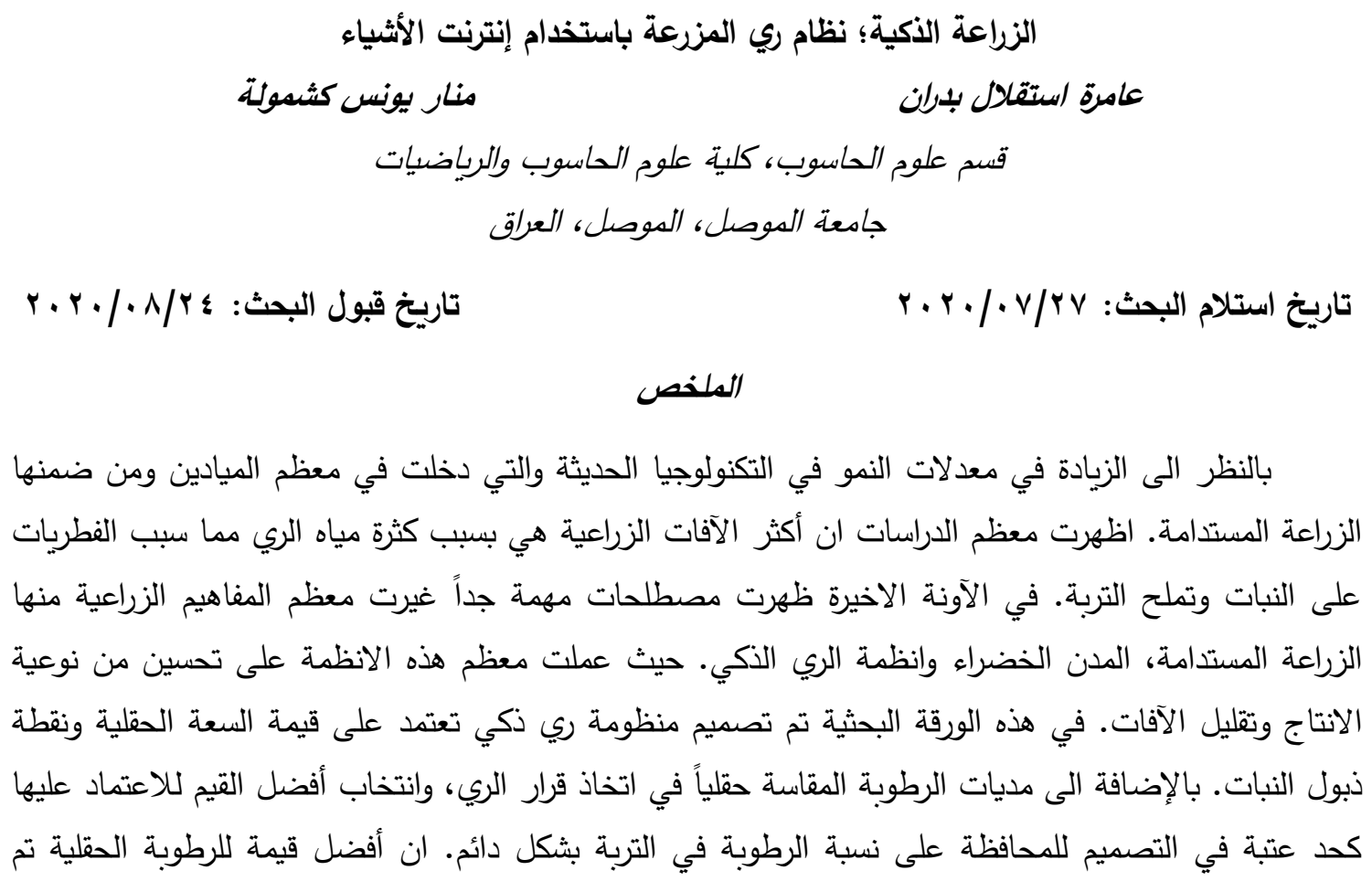




$$
\begin{aligned}
& \text { تسجلها عند التصميم كانت عند حد العتبة 24\% لحقل تربته طينية. اخيراً تم استخدام أفضل انواع المسيطرات } \\
& \text { ESP8266 \& ESP-32S } \\
& \text { Message Queuing Telemetry Transport (MQTT) } \\
& \text { المنظومة والحوسبة السحابية. } \\
& \text { الكلمات المفتاحية: انترنت الأثياء، بروتوكول MQTT، الزراعة الذكية، متحس الرطوبة، المحتوى الرطوبي، } \\
& \text { السعة الحقلية، نقطة الذبول. }
\end{aligned}
$$

\section{Introduction}

The term internet of things (IoT), which was formulated by Kevin Ashten in 1999, has become more common and more usable [1]. Internet of Things is a developed concept for the smart internet, which owns every materialistic thing in our life that can be reached by the internet or connect with each other in order to send and receive the data, to perform particular or general tasks by the network. For instance; all the modern devices and tools which are daily used are connected to the internet, and administered by mobile apps, computer or by other devices that are connected to the global network [2][3].

The internet of things gives human the capability to control things remotely without the need for existence in the same place or near a device. Such technologies are useful in smart houses, sensing and monitoring instruments that are connected to the internet. This controlling technology can remotely turn on and off the devices that are in touch with the life of human being, such as air-conditioning devices[1][7].

The internet of things can be viewed by the type of connecting things in it. The word "things" here refers to all the smart devices; such as TV, mobile phone, watches, glasses, and alarm and monitoring devices, and other devices that can be connected to each other through the internet. These devices can react with each other in the network without the intervention of human, or even the reaction of human with these devices through the network [12]. The development of protocols of the internet of things reveals the evolution of a new generation of protocols that contribute in the development of the internet of things [10]. Though most of us do not have smart houses that contain devices which talk to each other; the internet of things is greatly being developed nowadays. Many forms of connections, internet of things constituents and their protocols are imagined in a way that is beyond human ability in creation and invention. And this is the motive behind the great fast development of internet of things [9][14].

Modern technology has been applied in many fields of life including agriculture, when modern agriculture depended on the automatic systems that are controlled and monitored electronically along all the agricultural season. This technology contributed in increasing the production and improve it in addition to avoid waste of consumed water and protect the soil from salinity, the matter which will be reflected on the health of the plant. In comparison with the classic systems of agriculture; the smart agriculture proved supremacy in various fields such as increase of production and diseases resistance, as the previous studies referred to, which were compared with the proposed studies as follows:

In [5] a low-cost system for monitoring the farm was designed, and achieved according the applied type for measuring moisture ranges in field soil continuously. The farmer was informed with the low moisture content by text messages and expose the results according to temporal sequence on the Losant platform. In comparison with the designed sample it is noticed that field capacity, and wilting point were taken into 
account, and through both of them the threshold value was calculated, in addition to moisture ranges which raises the efficiency of agricultural field.

In [6] cloud algorithms were provided to the smart irrigation system where all sensor devices and the microcontrollers that are used in building those systems were described. As in [13], a new vision was presented to monitor the soil moisture content in addition to temperature at the same time; Water Mark was also used to determine the location of waters in the field. On the other hand; the appropriate temperature degrees for each plant were secured by using the highest standards of accuracy in sensors which were connected to the sequential port to increase accuracy. Also, the best sophisticated microcontrollers ESP-32S were used in wilting point to increase the speed in uploading and make the decision of irrigation. Finally, relying on water properties in making the irrigation decision to ensure quality in irrigation.

In this paper, an expandable smart irrigation system was designed by adding new elements the farmer may see them necessary for the agricultural field. An algorithm was implemented to select the best moisture value for the plant by using field capacity factor and wilting point as basic conditions for calculating irrigation threshold value. All parts of the system may work separately or together in order to secure and upload moisture data to one of the monitoring Adafruit servers via the best fast protocols dedicated to this process, which is MQTT. The latter is regarded as one of the pioneering protocols in transferring both light and heavy data at the same time. It was designed to deal particularly with the Internet of Things. This system was programmed by Micro $\mathrm{C}$ using the Arduino IDE program. The software was then uploaded to the ESP board via the USB port integrated with this board.

\section{Proposal and Solution}

This research was achieved depending on the internet of things to design a smart irrigation system. And the highest rates of moisture levels were achieved by using the agricultural constants in the design. The most common problems of the electronic circuits are those used in agricultural fields, such as the difficulty of supplying all parts of the field with the required electric power. A single agricultural field has a number of nodes that collect data from the field. The most apparent problem of the smart systems is how to manage the huge amount of data in real time, and control it in decision making in addition to modeling it for monitoring.

\subsection{Electrical Connection and Communications Insurance}

The client unit is considered the basic brick in designing this system, because it is characterized with an electric connection independent from the controlling center in the Adafruit server by MQTT protocol. In other words; it is a complete independent unit works alone for it contains power resource. This resource consists of a solar cell works to generate the required energy to operate the electronic circuit, along with a charger and a battery used for storing the operating power during night or in case of emergency. This unit also can work separately, without the main Adafruit server. The microcontroller ESP8266 NodeMCU has special algorithm to select the best value for the moisture content in the event of an internet service, and interruption which can be used in case of disconnection with the main Adafruit server. As shown in Figure (1), client circuit describes how the client unit is built. This unit consists of the microcontroller ESP8266 NodeMCU which is the heart of this unit, as well as soil moisture sensor which is responsible for communication between the microcontroller and the state of moisture. This sensor was connected with the Analog input (A0) to 
increase the accuracy of this sensor and supply it with $(3.3 \mathrm{~V})$ electric power. Also some important data are displayed on the a screen attached type 0.96 Organic LightEmitting Diode (OLED) to the client unit works by a four pole Inter-Integrated circuit $\left(\mathrm{I}^{2} \mathrm{C}\right)$ protocol, two of which are to provide the screen with power (VCC.GND) and the others are connected with the digital entrance of the microcontroller (D1.D3) successively. The client unit measures the moisture of the soil and uploads it with a Node ID to the access point, then to Adafruit server through the gateway unit. In addition to operate the water pump that is connected with it in case this is required; and this is done by connecting it with one of the digital exit. Finally; the client unit is connected with the gateway unit by means of Wi-Fi connection and the gateway becomes the main server of the field in this period.

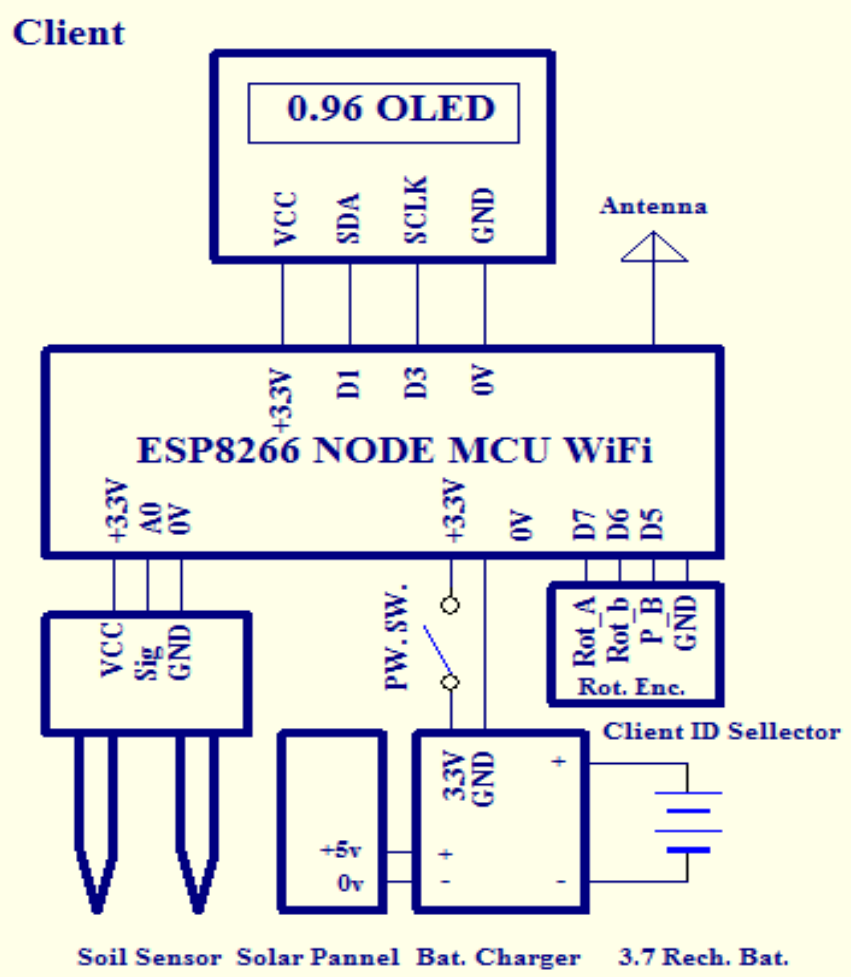

Figure (1) Design of the electronic circuit of the client unit by the microcontroller ESP8266 NodeMCU

\subsection{Access Point Design by Microcontroller ESP-32S NodeMCU}

After designing the access point which is necessary for connecting the far nodes of the field; the access point works as a link between the nodes around the field and the node in the center on one side, and the gateway unit on the other. Then it collects data from the nodes that are gained by the sensor that is connected with the node and with Node ID in the field. The access point schedules the data that comes from each node separately along with the Node ID. Then they are stored in a temporary store then to be prepared and framed uniformly. The access point was designed and programmed on the microcontroller ESP-32S NodeMCU and enables it to connect with the rest of the nodes. It is worth mentioning that the microcontroller ESP-32S NodeMCU has the Soft A.P specificity which was exploited in the design. This specificity makes the processor connects with two points wirelessly; which means that it works as a wireless bridge between any two devices, and the data is named by through this bridge. But there are 
some challenges in this case; it can deal with maximum 8 TCP connection which means that it can deal with 8 devices at one time. But in this research 5 devices only were used taking into consideration its frequency $(160 \mathrm{MHz})$ and the rate of transference speed 9600 baud rate when sending and receiving.

When devices are operated; the access point prepares connection with the other nodes. Also, the values of moisture in the sensors that are connected to the nodes must be checked. The access point also prepares the screen type 1.44 Thin Film Transistor (TFT) which is connected to the access point. The security of the data is necessary; therefore, an implicit password was created so the data that comes from the node are not subject to spoil. Among the main tasks of the access point is to keep connection with the gateway unit through the serial port in order to transfer greater and faster data at the same time. The serial exit of the access point was connected with the exit between the two numbers (D16 which is devoted to receiving data) and (D17 which is devoted to sending data). The access point controls the data that comes from the nodes and displays some data of these nodes such as the IP and the MAC address. In addition to the data of moisture values and the Node ID. Finally the data are framed with one frame and sent to the gateway unit like masses of data then to be stored and scheduled within this unit as it is shown in figure (2).

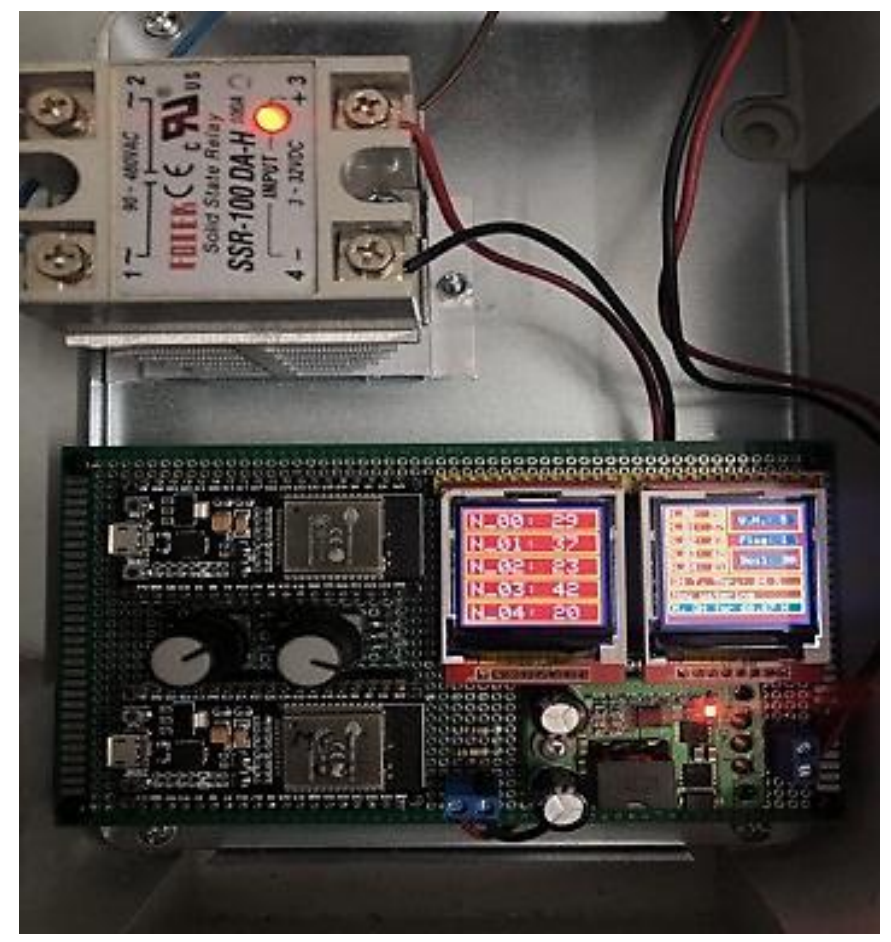

Figure (2) the access point and the gateway unit in operation

\subsection{Gateway Unit Design Using micro-Controller ESP-32S NodeMCU}

The design is considered the heart of irrigation system and the basic foundation that the rest of the system, is based on and it is the main center of the decision and the controller of all the nodes. In addition it is the machine that works on transferring data among the departments of the system when it works. It is the link between the internet on the one side, and the irrigation system that consist of microcontrollers and sensors on the other. The gateway unit was designed by using the microcontroller ESP-32S NodeMCU, in order to connect the internet with the access point directly and with the 
nodes of the field, to measuring and uploading the levels of soil moisture. The gateway unit in the beginning prepares the path and maintain Adafruit server, via internet to contact the server. This contact is for updating data temporally taking into consideration that the system works in real time. The data that are obtained from the nodes of the field are scheduled through the access points. Then they pass to the gateway unit and to be put in a table in the EEPROM memory of the gateway unit particularly edited to make use of in making a decision or predicting the new values. Also to display data on a screen successively 1.4 TFT color screen: this unit prepares the water pump and operates it if required after taking the appropriate decision. The gateway unit is provided by electric power through separate resource away from the power resource of the nodes, as shown in figure 2 .

\subsection{Upload Data to the Adafruit Server and Make a Decision}

The client unit uploads the data of soil moisture with a Node ID to be scheduled successively in this unit before sending. At the beginning of the applied algorithm the connection between the gateway unit is secured through making the microcontroller ESP8266 NodeMCU in loop case that it cannot get out of, only in case of connection, and this loop is repeated every $0.5 \mathrm{sec}$. If the connection is not achieved; the microcontroller restarts. The connection is to be checked and repaired every $1 \mathrm{sec}$. And this unit is given IPv4 type Static IP, to remain with this IP in Adafruit server. In order to avoid losing the connection as a result of changing IPs from time to time when using Dynamic Host Configuration Protocol (DHCP) protocol. In case of achieving connection between the client unit and gateway unit through the access point representing the Adafruit server; the suitable speed of sending and receiving are restricted to 9600 baud rate, and restricts the number of the exit port no $=80$. This unit uploads the Node ID periodically along with the state of moisture in order to be scheduled in the Adafruit server and update the data every $0.5 \mathrm{sec}$. This unit can make some decisions through the objective algorithm in it if the server fails, to deliver the necessary data to the client unit to execute the decision of Adafruit server.

\subsection{Quantities of Rated Drip Irrigation Based on Soil Constants}

The modern methods in agriculture and irrigation technologies greatly contributed in increasing the production of crops, and enhanced the strength of many types of plants; and the best instances for such thing are smart irrigation systems and the sustainable green cities. All of this was achieved by virtue of the modern technologies that are applied in the agricultural sector generally. In This paper based on the subject of smart irrigation system using the threshold value in order to keep the rate of soil moisture permanently during all the seasons of the year. The calculation of threshold value is done by the following equation [11][2]:

A. $W=M_{f . c}-W . P$

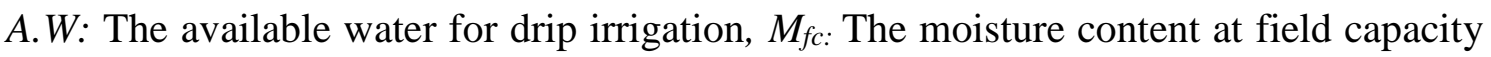
(\%), W.P: Wilting point of the plant

The value of the available water which was gained from the previous equation can be relied on in calculating threshold value after consuming $50 \%$ of the available water $\left(\mathrm{A} . \mathrm{W}^{*} 0.5\right)$. Threshold value was calculated by the following equation [3]:

Threshold value $=M_{f . c}-(A . W \times 0.5)$

The value of moisture content should not be less than threshold value; and if it is less the pump will be operated and by this method, we insure an appropriate moisture 
content during all the time of plant growth. The gained values are scheduled and printed in Adafruit server in order to refer to them when needed to notify the person who is responsible for monitoring if any defect in the smart irrigation system occurs.

\section{Decision of Irrigation in the Smart Irrigation System}

As it was mentioned above; irrigation decisions are taken depending on the results which are gained from the nodes. Irrigation decision depends on a stable value to be put in the system which is threshold value. Threshold value is calculated depending on agricultural constants which are Field Capacity F.C and Wilting Point W.P of the plant. These constants are used to apply the equations inside and outside gate unit. On this basis the threshold value changes from field to another.

\subsection{Irrigation Decision Based on Threshold Value}

This type of irrigation depends on threshold value that was calculated in the irrigation system considering F.C value as 0.32. And W.P value is 0.16. And through these two values the value of the available water is calculated through equation (1). Then the available water value, which was gained previously, is used to calculate threshold value after consuming $50 \%$ of the available water through equation (2). If the moisture content was less or equals threshold value; the pump will be operated for irrigation as in figure (3-A). When the values of moisture content rise higher than threshold value; the pump will be turned off automatically as in figure (3-B). In addition to increase the numbers of the nodes that raise the moisture content to select the best value as threshold value. It was found that the best value was $24 \%$ which was uploaded to Adafruit server.

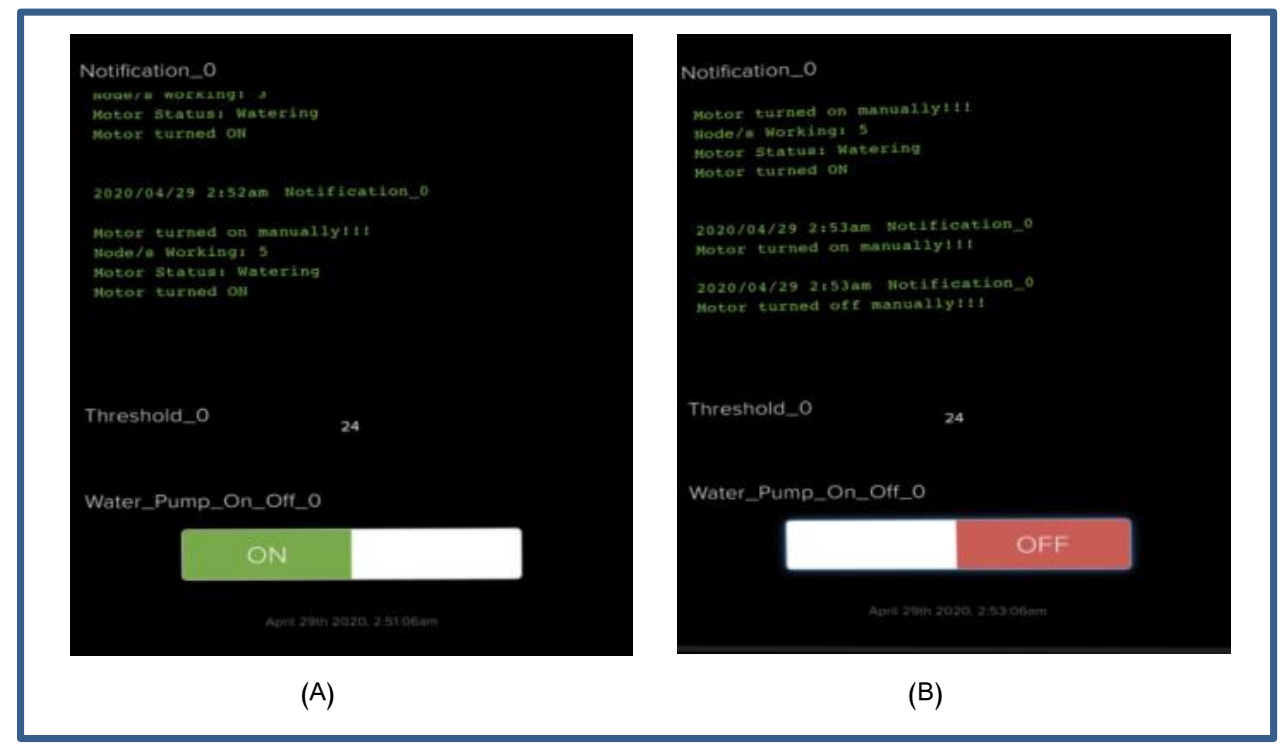

Figure (3) Taking decision on threshold value in the smart irrigation system

When irrigation process is completed; a gradual raise in the rate of moisture content that results from operating the pump is noticed, as in figure (4). 


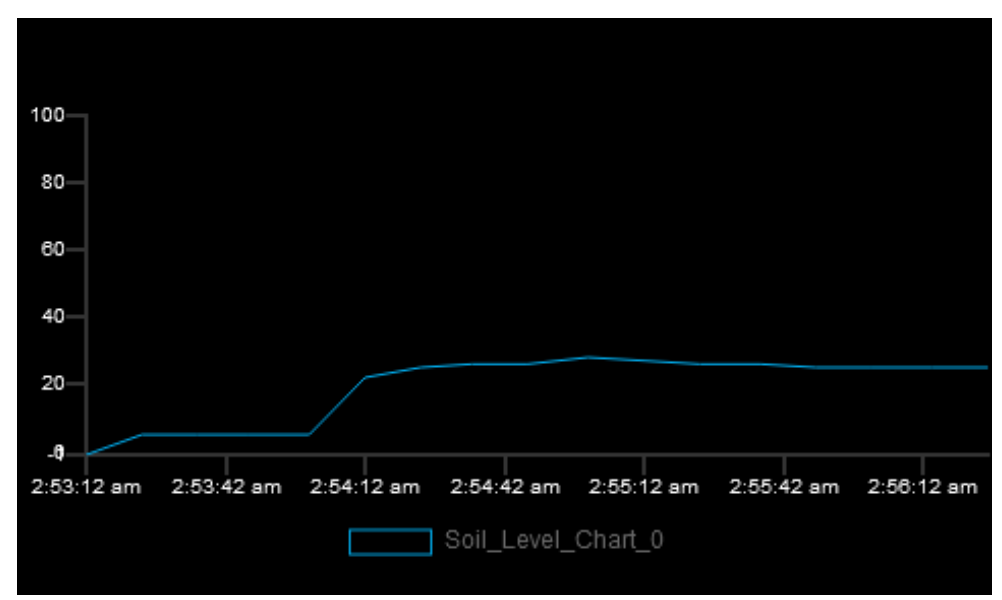

Figure (4) Monitoring the moisture content of the field by the Adafruit server

\section{Conclusions:}

Most of the recent studies concerning smart irrigation systems reached at the fact that quantities of water that are given to the plants, should be rationalized. Much quantities of water cause over salinity of soil and lead to consume the elements that are necessary for plant growth. From this point emerged the need for developing a smart irrigation system depends on the rate of field moisture levels as a basis to take the decision of irrigation when actually needed. The client unit was designed to be appropriate for working in the field for long periods of time in order to raise the rates of moisture continuously; without the need for any wireless connection provided with data resource, in addition to depending on the Field Capacity(0.32) and Wilting Point(0.16) of the plant for the benefit of threshold value $\% 24$ in the irrigation process. Also, the necessary power for operating the nodes of the client were provided from solar boards and compact batteries together in order to conform a complete unit not to be connected wirelessly with any source of power. 


\section{$\underline{\text { REFERENCES }}$}

[1] Bassi, A., Bauer, M., Fiedler, M., Kramp T., Kranenburg R., Lange, S. \& Meissner, S. (2013). "Enabling Things to Talk: Designing IoT solutions with the IoT Architectural Reference Model". Springer Heidelberg New York Dordrecht London.

[2] Dabbagh, M., \& Rayes, A. (2019). "Internet of things security and privacy". In Internet of Things From Hype to Reality (pp. 211-238). Springer, Cham.

[3] Farhan, L., Kharel, R., Kaiwartya, O., Quiroz-Castellanos, M., Alissa, A., \& Abdulsalam, M. (2018). "A concise review on Internet of Things (IoT)problems, challenges and opportunities". International Symposium on Communication Systems, Networks \& Digital Signal Processing (CSNDSP) (pp. 1-6). IEEE.

[4] Huntington, T. G. (2005). "Available water capacity and soil organic matter". In Encyclopedia of Soil Science-Two-Volume Set (pp. 139-143). CRC Press.

[5] Kodali, R.K., \& Sahu, A. (2016, December). "An IoT based soil moisture monitoring on Losant platform". In 2016 2nd International Conference on Contemporary Computing and Informatics (IC3I) (pp. 764-768). IEEE.

[6] Kokkonis, G., Kontogiannis, S., \& Tomtsis, D. (2017). "A smart IoT fuzzy irrigation system". Power (mW), 100(63), 25.

[7] Minerva, R., Biru, A., \& Rotondi, D. (2015). "Towards a definition of the Internet of Things (IoT)". IEEE Internet Initiative, 1, 1-86.

[8] Ottoni Filho, T. B., Ottoni, M. V., Oliveira, M. B. D., Macedo, J. R. D., \& Reichardt, K. (2014). "Revisiting Field Capacity (FC): variation of definition of FC and its estimation from pedotransfer functions". Revista Brasileira de Ciência do Solo, 38(6), 1750-1764.

[9] Perwej, Y., Haq, K., Parwej, F., Mumdouh, M., \& Hassan, M. (2019). "The Internet of Things (IoT) and its Application Domains". International Journal of Computer Applications, 975, 8887. 182(49).

[10] Ponnusamy, K., \& Rajagopalan, N. (2018). "Internet of Things: A Survey on IoT Protocol Standards". In Progress in Advanced Computing and Intelligent Engineering (pp. 651-663). Springer, Singapore.

[11] Tolk, J.A., (2003). "Soils, permanent wilting points". Encyclopedia Water Sci.. United States Department of Agriculture (USDA), Bushland, Texas, U.S.A., Marcel Dekker Inc.

[12] Uckelmann, D., Harrison, M., \& Michahelles, F. (Eds.). (2011). "Architecting the internet of things". Springer Science \& Business Media.

[13] Vellidis, G., Tucker, M., Perry, C., Kvien, C., \& Bednarz, C. (2008). "A realtime wireless smart sensor array for scheduling irrigation". Computers and electronics in agriculture, 61(1), 44-50.

[14] Vernesan, O., Friess, P., Woysch, G., Guillemin, P., Gusmeroli, S., Sundmaeker, H., Bassi, A., Eisenhauer, M. and Moessner, K., (2012). "The Internet of Things 2012: New Horizons". In IERC - Internet of Things European Research Cluster, ISBN 978-0-9553707-9-3, Halifax, UK, 2012. 